\title{
Analysis of sex-related differences in external load demands on beach handball
}

\section{Análise das diferenças relacionadas ao sexo nas demandas de carga externa no handebol de praia}

Carlos David Gómez-Carmona ${ }^{1}$

(D) http://orcid.org/0000-0002-4084-8124

David García-Santos ${ }^{1}$

(D) https://orcid.org/0000-0002-9952-7536

David Mancha-Triguero ${ }^{1}$

(D) https://orcid.org/0000-0001-8080-7565

Antonio Antúnez ${ }^{1}$

(D) https://orcid.org/0000-0002-7451-4448

Sergio José Ibáñez

(D) https://orcid.org/0000-0001-6009-4086
Abstract - The purpose of the present study was to analyze the sex-related differences in beach handball workload. A total sample of 125 handballers (male, $n=60$; female, $n=65$ ) belonging to six U'16 teams, seven U'18 teams and eight senior teams were monitored in the final round of 2018-2019 beach handball tournament celebrated during 3-days congested-fixture design. The external load variables Steps, Jumps, Player Load, Total Impacts (>2G) and Total Impacts per Intensities (very low, 2-4G; low, 4-6G; moderate, 6-8G; high, 8-10G; very high, >10G) through WIMU ${ }^{\mathrm{TM}}$ inertial devices were registered. Statistical analysis was composed by t-test and Cohen's $d$ for anthropometrical variables and by MANOVA and omega partial square for sex and categories related differences. Greater values in male handballers were found in height, weight and age in each categories (U'16: $\mathrm{p}<0.05$; $\mathrm{d}=0.50-2.26$; U'18: $\mathrm{p}<0.05 ; \mathrm{d}=0.95-2.21$; senior: $\mathrm{p}<0.05 ; \mathrm{d}=1.01-1.99)$, except in age in $\mathrm{U}^{\prime} 18(\mathrm{p}=0.97 ; \mathrm{d}=0.01)$. Respect to external workload, differences were found related to category $\left(\mathrm{p}<0.01 ; \omega_{\mathrm{p}}{ }^{2}=0.02-0.05\right.$, small $)$ and $\operatorname{sex}\left(\mathrm{p}<0.01 ; \omega_{\mathrm{p}}{ }^{2}=\right.$ $0.04-0.21$, small to high), except in Steps $\left(\mathrm{p}=0.47 ; \omega^{\mathrm{p}}=0.00\right)$, finding the greatest sex-related differences in U'16 category. From the differences found in anthropometrical characteristics and external workload, their evaluation during competition allows designing specific training sessions with the purpose of sports performance enhancement in beach handball.

Key words: Microtechnology; Workload; Sports; Youth sports.

Resumo - Objetivou-se analisar as diferenças relacionadas ao sexo na carga de trabalho de handebol de praia. Uma amostra total de 125 jogadores de handebol (masculino, $n=60$; feminino, $n=65$ ) pertencentes a seis equipes sub-16, sete equipes sub-18 e oito equipes seniores foi monitorada na rodada final do torneio de handebol de praia 2018-2019 durante 3 dias de projeto. As variáveis de carga externa Etapas, Saltos, Carga do jogador, Impactos totais (> 2G) e Impactos totais por intensidade (muito baixo, 2-4G; baixo, 4-6G; moderado, 6-8G; alto, 8-10G; muito alto, > 10G) foram mensurados por meio de dispositivos inerciais WIMU ${ }^{T M}$. A análise estatística foi composta pelo teste $t, d$ de Cohen e MANOVA. Maiores valores nos handebolistas masculinos foram encontrados em estatura, massa corporal e idade em cada categoria (U'16: $p<0,05 ; d=0,50-2,26$; $U$ '18: $p<0,05 ; d=0,95-2,21$; sénior: $p<0,05 ; d=1,01-1,99)$, exceto na idade nos $U^{\prime} 18(p=0,97 ; d=0,01)$. No que diz respeito à carga de trabalho externa, foram encontradas diferenças relacionadas à categoria $\left(p<0,01 ; \omega p^{2}=\right.$ $0,02-0,05$, pequeno) e sexo $\left(p<0,01 ; \omega p^{2}=0,04-0,21\right.$, pequeno a alto), exceto nas etapas $(p=0,47$; $\left.\omega p^{2}=0,00\right)$, encontrando as maiores diferenças relacionadas ao sexo na categoria U'16. A partir das diferenças encontradas nas características antropométricas e na carga de trabalho externa, sua avaliação durante a competição permite projetar sessões de treinamento especificas com o objetivo de melhorar o desempen ho esportivo no handebol de praia.

Palavras-chave: Microtecnologia; Carga de Trabalho; Esportes; Esportes Juvenis.
1 Department of Didactics of Body Expression, Plastic and Music. Faculty of Sports Sciences. University of Extremadura. Cáceres, Spain.

Received: February 12, 2020 Accepted: June 17, 2020

How to cite this article Gómez-Carmona CD, García-Santos D, Mancha-Triguero D, Antúnez A, Ibáñez SJ. Analysis of sex-related differences in external load demands on beach handball. Rev Bras Cineantropom Desempenho Hum 2020, 22:e71448. DOl: http://dx.doi. org/10.1590/1980-0037.2020v22e71448

Copyright: This work is licensed under a Creative Commons Attribution 4.0 International License. 


\section{INTRODUCTION}

Beach handball has been characterized as a relatively recent sport that is practiced in the summer season ${ }^{1}$. It is a worldwide recognized sport that appeared in the nineties, with its own regulations and federative organization $^{2}$. This sport modality presents great differences with the handball on the indoor court ${ }^{3}$. The beach handball is played on a sand surface of $27 \times 12$ meters, where each team is composed of three players and a goalkeeper and the duration of the match is divided into two halves of 10 minutes $^{4}$. Player substitutions are made throughout the sideband. The dynamics of substitutions allow teams to be in attack phase with one more player (simple numerical superiority, 4vs3), changing the goalkeeper by an attack specialist. Once the attack phase is finished, the specialist attack player is substituted by the goalkeeper in the defensive phase. For this fact, there is a double value of the goals when they are scored by the goalkeeper and after a $360^{\circ}$ turn or fly performed by any player (reception of the last pass in the air $)^{5}$. This fact causes it to be a very attractive, unpredictable and spectacular sport in relation to offensive technical actions ${ }^{6}$. If the match ends in a draw, the so-called "golden goal" is carried out to determine which team wins the match ${ }^{7}$.

The match dynamic with successively attack-defense cycles has an impact on the physical demands through the performance of numerous high-intensity actions in the most decisive key situations (throws, stops, counterattacks) ${ }^{8}$. These physical demands have a direct effect on physiological demands ${ }^{9,10}$. These high-intensity actions are combined with low-intensity activity periods in relation to the searching of free-spaces to score a goal or during the lateral displacements performed in the defensive phase. This fact is produced by the reduced dimensions of the field of play so that it is not necessary to perform long high-intensity displacements s,7,8. $^{5}$. These specific physical-technical-tactical characteristics of beach handball make it necessary the analysis of physical and physiological demands during the competition with the aim to design specific training sessions for performance enhancement ${ }^{10}$.

Different technologies have been developed for monitoring and quantify the workload demands during the competition ${ }^{11}$, for both internal load $^{12}$ and external load through time-motion analysis on indoor ${ }^{13}$ and outdoor conditions ${ }^{14}$, tactical analysis ${ }^{15}$ and neuromuscular load through accelerometry ${ }^{16}$ on an accurate and reliable way.

The monitoring through electronic performance and tracking systems (EPTS) is very extended in handball through optical systems and inertial devices $^{17,18}$. In beach handball, only two previous studies realized by Pueo et al. ${ }^{8}$ and Zapardiel and Asín-Izquierdo ${ }^{19}$ have investigated the workload demands in competition. These studies have shown that players covered a total distance between 600 and 1200 meters, being most of them low-intensity displacements $(1-12 \mathrm{~km} / \mathrm{h})$, with low-intensity impacts $(5-6 \mathrm{G})$ and moderate physiological demands $\left(71-80 \% \mathrm{HR}_{\mathrm{MAX}}\right)$. Another important 
aspect that needs to be considered is the players' gender, due to significative differences have been found both in handball and beach handball. Female and male players performed equal volume of demands (displacements, impacts, accelerations, and decelerations) while male players performed these action with higher intensity (high-intensity action, sprinting displacements, maximum accelerations and decelerations, and severe impacts/collisions) ${ }^{8,19}$.

Therefore, related to the lack of research that quantify the workload demands in beach handball at elite-level in senior players and in youth players, and the gender-related differences with the purpose of design individualized training sessions to performance enhancement, the purpose of the present study were to describe the anthropometrical and physical profile of beach handball players and to compare these variables between genders (male and female) and players category (under-16, under-18 and senior) that have been registered through inertial measurement units.

\section{METHODS}

\section{Participants}

The study sample was composed of 125 players, including 60 men and 65 women. The age of the participants was between 13 and 28 years. The teams that participated were six under-16 teams $(n=30)$, seven under-18 teams $(n=35)$ and eight senior teams (over 18 years old) $(n=40)$. The anthropometric characteristics of the participants are shown in Table 1.

The approval of the Bioethics Committee of the University of Extremadura (registration code 67/2017) was obtained, following the ethical guidelines of the Declaration of Helsinki. Before the tournament, all subjects were informed about the characteristics of the study, its procedures, the benefit of the results and the possible risks associated with the participation of the study associated with the sports practice. All participants signed the informed consent, and their legal guardians also signed the informed consent for the under-18 players.

\section{Instruments}

\section{- Anthropometric characteristics}

Age, height, weight and body-mass index variables were registered in a questionnaire paper. Height was measured through a rod stadiometer (SECA, Hamburg, Germany) and weight was measured through a portable body composition monitor (model BC-601, TANITA, Tokio, Japan).

\section{- External load analysis}

For registering workload demands during the tournament, all players were equipped with inertial measurement units (IMUs) called WIMUTM (RealTrack Systems, Almeria, Spain). This device included multiple sensors (accelerometers, gyroscopes, magnetometers, and a global navigation satellite system sensor). Only variables obtained through the sensorial fusion of the four accelerometers that composed the device were used. 
The accelerometers have a full scale of $\pm 16, \pm 16, \pm 32$ and $\pm 400 \mathrm{G}$ and were set with a sampling frequency of $100 \mathrm{~Hz}$ (100 data per second). This sensor has reported very good reliability in laboratory and field conditions ${ }^{16}$.

The IMUs were attached to players through an anatomically adjusted vest at the interscapular line (T2-T4 vertebra) to not producing movement during registration, and have been utilized previously in different investigations performed in handball with players and referees ${ }^{20,21}$.

The external workload variables analyzed in the present study are shown on Box 1, which were relativized to playing time (per minute): (a) total impacts; (b) low intensity impacts; (c) moderate-intensity impacts; (d) high-intensity impacts; (e) very-high-intensity impacts; (f) severe intensity impacts d; (g) player load; (h) steps; and (i) jumps.

Box 1. External workload variables registered in the present study.

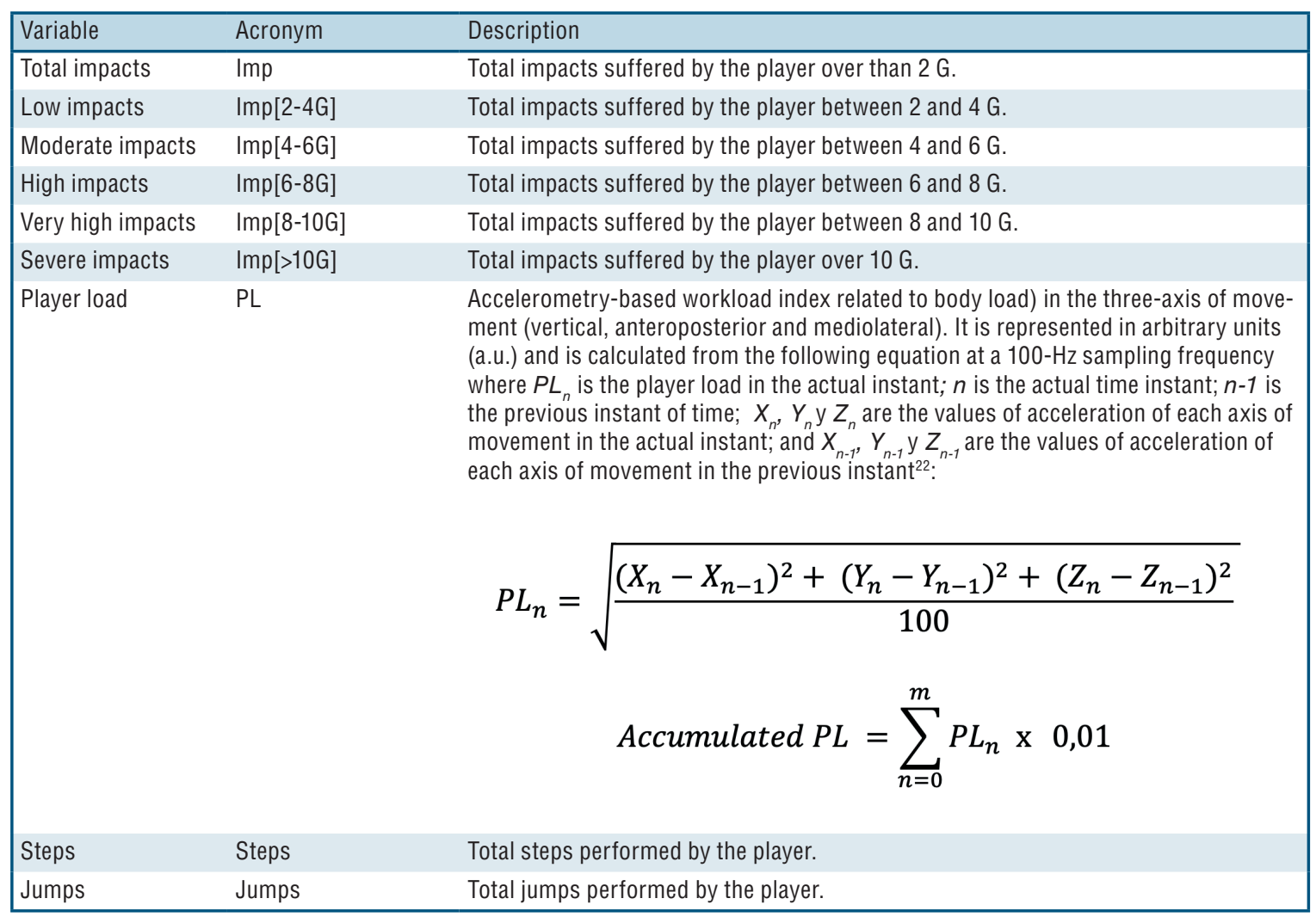

\section{- Independent variables}

The analyzed independent variables in the present study were gender and players category, showing the sample size of each group that was analyzed.

a) Gender: All the participants were distributed in two groups: 60 male athletes $(n=166)$ y 65 female athletes $(n=162)$.

b) Category: According to participants' age they were distributed in three categories: (a) under 16, 30 players between 14 and 15 years distributed in six teams $(n=113)$; (b) under 18, 30 players between 16 
and 17 distributed in seven teams $(n=100)$; (c) senior, 45 players older than 18 years distributed in eigth teams $(n=115)$.

\section{Procedures}

The tournament had a duration of three days, from July $19^{\text {th }}$ to July $21^{\text {st }}$ during 2019. All the participants' teams in under-18 and senior categories were divided into two groups, where teams played in a league format (all against all one time). At the end of the group stage, the best eight teams qualified for the quarter-finals. The teams that won the quarter-finals qualified to semifinals. The winner teams in the semifinal played the final of the tournament, while the loser teams played the third and fourth place. Instead, due to the under-16 category participated five male and three female teams, only a league format (all against all one time) was played. The competition was performed on Friday between 9 a.m. and 11 p.m. While, the matches of the under-18 and senior categories were played on Saturday between 9 a.m. and 11 p.m., and on Sunday between 10 a.m. and 2 p.m.

A total of 84 matches were played during the tournament of which 60 matches were registered. Before the start of the tournament, anthropometrical characteristics of handballers were measured. Then, 15 minutes before the matches, the players were cited to attach the inertial devices and vests. At the end of the matches, the inertial devices and vests were removed from the players and data was downloaded and analyzed in a laptop with the software SPRO ${ }^{\mathrm{TM}}$ (RealTrack Systems, Almeria, Spain). The criteria to include players' data, in the final analysis, was to participate more than $30 \%$ of total playing time in each half. This criterion was employed for homogeneity of the players' participation in subsequent analysis, due to the differentiated attack-defense roles only allow play a $50 \%$ of total playing time ${ }^{5}$.

\section{Statistical analysis}

Firstly, an exploratory analysis by criteria assumption test was performed: (a) normality (Shapiro-Wilk test), and (b) homoscedasticity (Levene test), obtaining all of them a normal distribution ${ }^{23}$. Then, a descriptive analysis was realized, showing data as mean and standard deviation $(\mathrm{M} \pm \mathrm{SD})$ to describe the external workload demands in the analyzed matches during the tournament in function of the gender in each players' category. For calculating the average of demands in each match, the analyzed variables were relativized by playing time per minute. To compare the differences of anthropometric characteristics between genders, an independent samples t-test was performed. Instead, to compare the external workload related to gender and players' category, a MANOVA test was realized. The statistical significance was established as $p<0.05$.

To calculate the magnitude of differences for independent samples t-test was utilized Cohen's d $(d)$, while for MANOVA test was utilized partial omega squared $\left(\omega_{p}^{2}\right)$. Cohen's d $(d)$ was interpreted as: trivial (0-0.2), low (0.2-0.6), moderate (0.6-1.2), high (1.2-2.0) and very high (>2.0 $)^{24}$. Instead, 
omega partial squared $\left(\omega_{p}{ }^{2}\right)$ was interpreted following Cohen ${ }^{24}$ : $>0.01$ low; $>0.06$ moderate and $>0.14$ high. Statistical analyses were performed by the software Statistical Package of Social Science (version 24, 2016; IBM Corp., Armonk NY, USA).

\section{RESULTS}

In Table 1 is shown the descriptive analysis of the players' anthropometrical characteristics and the comparison analysis between genders in each age group. Statistical differences were found in all age groups in weight and height with higher values in males with a moderate to very high effect size. Also, statistical differences in age were found with older values in males, except in the under-18 category.

The external workload demands registered during the tournament related to the gender and age category are shown in Table 2. Genderrelated differences were found with a moderate to high effect size in all age groups, while the effect of players' category was low. In the pairwise comparison, the highest values were found in the under-16 male category, which presents the greatest differences with the under-16 female category.

Table 1. Descriptive and inferential analysis of anthropometrical characteristics of analyzed participants related to age category

\begin{tabular}{|c|c|c|c|c|c|c|c|}
\hline & Variables & $\begin{array}{l}\text { Male } \\
\mathrm{M} \pm \mathrm{SD} \\
(95 \% \mathrm{Cl})\end{array}$ & $\begin{array}{l}\text { Female } \\
\mathrm{M} \pm \mathrm{SD} \\
(95 \% \mathrm{Cl})\end{array}$ & $\begin{array}{l}\text { Total } \\
\mathrm{M} \pm \mathrm{SD} \\
(95 \% \mathrm{Cl})\end{array}$ & $p$ & $d$ & effect \\
\hline \multirow{4}{*}{ Under-16 } & $\begin{array}{l}\text { Age } \\
\text { (years) }\end{array}$ & $\begin{array}{l}15.79 \pm 0.42 \\
(15.56-16.02)\end{array}$ & $\begin{array}{l}15.42 \pm 0.92 \\
(14.91-15.92)\end{array}$ & $\begin{array}{l}15.60 \pm 0.73 \\
(15.33-15.87)\end{array}$ & $<.05$ & 0.50 & + \\
\hline & $\begin{array}{l}\text { Weight } \\
\text { (kg) }\end{array}$ & $\begin{array}{l}65.52 \pm 9.94 \\
(60.02-71.02)\end{array}$ & $\begin{array}{l}60.57 \pm 9.28 \\
(55.44-65.71)\end{array}$ & $\begin{array}{l}63.05 \pm 9.77 \\
(59.44-66.70)\end{array}$ & $<.05$ & 0.52 & + \\
\hline & $\begin{array}{l}\text { Height } \\
(\mathrm{cm})\end{array}$ & $\begin{array}{l}172.36 \pm 5.55 \\
(169.29-175.43)\end{array}$ & $\begin{array}{l}160.71 \pm 4.78 \\
(158.06-163.36)\end{array}$ & $\begin{array}{l}166.54 \pm 7.81 \\
(163.62-169.45)\end{array}$ & $<.01$ & 2.26 & ++++ \\
\hline & $\begin{array}{l}\mathrm{BMI} \\
\left(\mathrm{kg} / \mathrm{m}^{2}\right)\end{array}$ & $\begin{array}{l}22.01 \pm 2.92 \\
(20.39-23.63)\end{array}$ & $\begin{array}{l}23.53 \pm 4.04 \\
(21.29-25.77)\end{array}$ & $\begin{array}{l}22.77 \pm 3.55 \\
(21.44-24.10)\end{array}$ & .25 & -0.19 & \\
\hline \multirow{4}{*}{ Under-18 } & $\begin{array}{l}\text { Age } \\
\text { (years) }\end{array}$ & $\begin{array}{l}17.42 \pm 0.81 \\
(16.96-17.89)\end{array}$ & $\begin{array}{l}17.41 \pm 0.97 \\
(16.97-17.85)\end{array}$ & $\begin{array}{l}17.42 \pm 0.89 \\
(17.11-17.72)\end{array}$ & .97 & 0.01 & \\
\hline & $\begin{array}{l}\text { Weight } \\
(\mathrm{kg})\end{array}$ & $\begin{array}{l}71.57 \pm 15.47 \\
(62.64-80.50)\end{array}$ & $\begin{array}{l}60.13 \pm 8.28 \\
(56.36-63.90)\end{array}$ & $\begin{array}{l}64.17 \pm 12.81 \\
(60.30-69.11)\end{array}$ & $<.05$ & 0.95 & ++ \\
\hline & $\begin{array}{l}\text { Height } \\
\text { (cm) }\end{array}$ & $\begin{array}{l}178.00 \pm 4.39 \\
(175.47-180.53)\end{array}$ & $\begin{array}{l}165.24 \pm 6.72 \\
(162.18-168.30)\end{array}$ & $\begin{array}{l}170.34 \pm 8.61 \\
(167.38-173.30)\end{array}$ & $<.05$ & 2.21 & ++++ \\
\hline & $\begin{array}{l}\mathrm{BMI} \\
\left(\mathrm{kg} / \mathrm{m}^{2}\right)\end{array}$ & $\begin{array}{l}22.48 \pm 4.28 \\
(20.01-24.95)\end{array}$ & $\begin{array}{l}22.03 \pm 2.85 \\
(20.73-23.32)\end{array}$ & $\begin{array}{l}22.21 \pm 3.44 \\
(21.03-23.39)\end{array}$ & .71 & 0.12 & \\
\hline \multirow{4}{*}{ Senior } & $\begin{array}{l}\text { Age } \\
\text { (years) }\end{array}$ & $\begin{array}{l}23.92 \pm 5.00 \\
(21.65-26.20)\end{array}$ & $\begin{array}{l}19.54 \pm 3.72 \\
(17.75-21.33)\end{array}$ & $\begin{array}{l}21.84 \pm 4.91 \\
(20.27-23.41)\end{array}$ & $<.05$ & 1.01 & ++ \\
\hline & $\begin{array}{l}\text { Weight } \\
(\mathrm{kg})\end{array}$ & $\begin{array}{l}80.71 \pm 13.83 \\
(74.41-87.00)\end{array}$ & $\begin{array}{l}58.65 \pm 8.46 \\
(54.58-62.73)\end{array}$ & $\begin{array}{l}70.23 \pm 15.99 \\
(65.12-75.34)\end{array}$ & $<.05$ & 1.97 & +++ \\
\hline & $\begin{array}{l}\text { Height } \\
(\mathrm{cm})\end{array}$ & $\begin{array}{l}179.95 \pm 7.56 \\
(176.51-183.39)\end{array}$ & $\begin{array}{l}166.05 \pm 6.45 \\
(162.94-169.16)\end{array}$ & $\begin{array}{l}173.35 \pm 9.90 \\
(170.19-176.51)\end{array}$ & $<.05$ & 1.99 & +++ \\
\hline & $\begin{array}{l}\mathrm{BMI} \\
\left(\mathrm{kg} / \mathrm{m}^{2}\right)\end{array}$ & $\begin{array}{l}24.84 \pm 3.44 \\
(23.28-26.41)\end{array}$ & $\begin{array}{l}21.27 \pm 2.78 \\
(19.93-22.61)\end{array}$ & $\begin{array}{l}23.14 \pm 3.59 \\
(21.99-24.29)\end{array}$ & $<.05$ & 1.15 & ++ \\
\hline
\end{tabular}

Note. M: mean; SD: standard deviation; Cl: confidence interval; p: p-value; d: Cohen’s d effect size (low "+"; moderate "++"; high "+++"; very high "++++". 
Table 2. Analysis of gender-related and category-related differences in the external workload performed by the participants in the present study.

\begin{tabular}{|c|c|c|c|c|c|}
\hline Variable & Category & $\begin{array}{l}\text { Male } \\
\mathrm{M} \pm \mathrm{SD} \\
(95 \% \mathrm{Cl})\end{array}$ & $\begin{array}{l}\text { Female } \\
\mathrm{M} \pm \mathrm{SD} \\
(95 \% \mathrm{Cl})\end{array}$ & $\begin{array}{l}\text { Sex } \\
F(p) \\
\omega_{p}^{2} \text { (effect) }\end{array}$ & $d$ (effect) \\
\hline \multirow{5}{*}{ Imp } & Under-16 & $\begin{array}{l}747.02 \pm 306.52 \\
(664.93-829.10)\end{array}$ & $\begin{array}{l}414.89 \pm 206.36 \\
(360.13-469.64)\end{array}$ & \multirow{3}{*}{$\begin{array}{l}42.09(<.001) \\
0.11 \text { (moderate) }\end{array}$} & 1.29 (high) \\
\hline & Under-18 & $\begin{array}{l}499.48 \pm 256.29 \\
(425.07-573.90)\end{array}$ & $\begin{array}{l}398.34 \pm 190.23 \\
(345.38-451.31)\end{array}$ & & 0.45 (low) \\
\hline & Senior & $\begin{array}{l}571.59 \pm 266.34 \\
(503.95-639.23)\end{array}$ & $\begin{array}{l}477.13 \pm 218.19 \\
(416.99-537.27)\end{array}$ & & 0.39 (low) \\
\hline & $\begin{array}{l}F(p) \\
\omega_{p}^{2} \text { (effect) }\end{array}$ & $\begin{array}{l}7.73(<.001) \\
0.04 \text { (low) }\end{array}$ & & $\begin{array}{l}\text { Interaction } \\
8.49(<.001)\end{array}$ & \\
\hline & post-hoc & $A, B$ & & 0.04 (low) & \\
\hline \multirow{5}{*}{$\operatorname{Imp}[2-4 \mathrm{G}]$} & Under-16 & $\begin{array}{l}575.19 \pm 240.10 \\
(510.89-639.23)\end{array}$ & $\begin{array}{l}336.60 \pm 147.91 \\
(297.35-375.85)\end{array}$ & \multirow{3}{*}{$\begin{array}{l}42.04 \text { (<.001) } \\
0.11 \text { (moderate) }\end{array}$} & 1.22 (high) \\
\hline & Under-18 & $\begin{array}{l}389.31 \pm 198.80 \\
(331.59-447.09)\end{array}$ & $\begin{array}{l}321.99 \pm 146.99 \\
(281.21-362.78)\end{array}$ & & 0.39 (low) \\
\hline & Senior & $\begin{array}{l}469.66 \pm 174.62 \\
(425.32-514.01)\end{array}$ & $\begin{array}{l}381.30 \pm 168.26 \\
(334.92-427.68)\end{array}$ & & 0.51 (low) \\
\hline & $\begin{array}{l}F(p) \\
\omega_{p}^{2} \text { (effect) }\end{array}$ & $\begin{array}{l}8.33(<.001) \\
0.04 \text { (low) }\end{array}$ & & $\begin{array}{l}\text { Interaction } \\
7.23(<.001)\end{array}$ & \\
\hline & post-hoc & $A, B$ & & 0.04 (low) & \\
\hline \multirow{5}{*}{$\operatorname{Imp}[4-6 G]$} & Under-16 & $\begin{array}{l}120.14 \pm 54.83 \\
(105.46-134.82)\end{array}$ & $\begin{array}{l}57.73 \pm 45.74 \\
(45.60-69.87)\end{array}$ & \multirow{3}{*}{$\begin{array}{l}38.93(<.001) \\
0.10 \text { (moderate) }\end{array}$} & 1.25 (high) \\
\hline & Under-18 & $\begin{array}{l}77.39 \pm 44.08 \\
(64.59-90.91)\end{array}$ & $\begin{array}{l}59.19 \pm 41.19 \\
(47.72-70.65)\end{array}$ & & 0.43 (low) \\
\hline & Senior & $\begin{array}{l}125.36 \pm 105.37 \\
(98.60-152.11)\end{array}$ & $\begin{array}{l}76.67 \pm 46.85 \\
(63.75-89.58)\end{array}$ & & 0.62 (moderate) \\
\hline & $\begin{array}{l}F(p) \\
\omega_{p}^{2} \text { (effect) }\end{array}$ & $\begin{array}{l}7.44(<.001) \\
0.04 \text { (low) }\end{array}$ & & $\begin{array}{l}\text { Interaction } \\
3.45(.033)\end{array}$ & \\
\hline & post-hoc & $A, C$ & & 0.01 (low) & \\
\hline \multirow{5}{*}{$\operatorname{Imp}[6-8 \mathrm{G}]$} & Under-16 & $\begin{array}{l}35.15 \pm 20.89 \\
(29.55-40.74)\end{array}$ & $\begin{array}{l}13.70 \pm 16.34 \\
(9.37-18.04)\end{array}$ & \multirow{3}{*}{$\begin{array}{l}88.82(<.001) \\
0.21 \text { (high) }\end{array}$} & 1.16 (moderate) \\
\hline & Under-18 & $\begin{array}{l}23.50 \pm 15.96 \\
(18.87-28.14)\end{array}$ & $\begin{array}{l}11.48 \pm 9.12 \\
(8.94-14.02)\end{array}$ & & 0.95 (moderate) \\
\hline & Senior & $\begin{array}{l}30.90 \pm 19.60 \\
(25.92-35.88)\end{array}$ & $\begin{array}{l}13.90 \pm 9.82 \\
(11.19-16.61)\end{array}$ & & 1.13 (moderate) \\
\hline & $\begin{array}{l}F(p) \\
\omega_{p}^{2}(\text { effect })\end{array}$ & $\begin{array}{l}5.12(.006) \\
0.02 \text { (low) }\end{array}$ & & \multirow{3}{*}{$\begin{array}{l}\text { Interaction } \\
2.27 \text { (.105) } \\
0.01 \text { (low) }\end{array}$} & \\
\hline & post-hoc & A & & & \\
\hline \multirow{5}{*}{$\operatorname{Imp}[8-10 \mathrm{G}]$} & Under-16 & $\begin{array}{l}10.56 \pm 8.23 \\
(8.36-12.76)\end{array}$ & $\begin{array}{l}4.19 \pm 6.88 \\
(2.37-6.02)\end{array}$ & & 0.85 (moderate) \\
\hline & Under-18 & $\begin{array}{l}6.05 \pm 4.85 \\
(4.65-7.46)\end{array}$ & $\begin{array}{l}3.23 \pm 3.41 \\
(2.28-4.18)\end{array}$ & \multirow[t]{2}{*}{$\begin{array}{l}47.70(<.001) \\
0.13 \text { (moderate) }\end{array}$} & 0.68 (moderate) \\
\hline & Senior & $\begin{array}{l}6.63 \pm 5.17 \\
(5.32-7.94)\end{array}$ & $\begin{array}{l}2.99 \pm 2.63 \\
(2.26-3.71)\end{array}$ & & 0.91 (moderate) \\
\hline & $\begin{array}{l}F(p) \\
\omega_{p}^{2} \text { (effect) }\end{array}$ & $\begin{array}{l}8.31(<.001) \\
0.04 \text { (low) }\end{array}$ & & \multirow{2}{*}{\multicolumn{2}{|c|}{$\begin{array}{l}\text { Interaction } \\
2.99 \text { (.051) } \\
0.01 \text { (low) }\end{array}$}} \\
\hline & post-hoc & $A, B$ & & & \\
\hline
\end{tabular}

Continue... 


\begin{tabular}{|c|c|c|c|c|c|}
\hline Variable & Category & $\begin{array}{l}\text { Male } \\
M \pm S D \\
(95 \% \mathrm{Cl})\end{array}$ & $\begin{array}{l}\text { Female } \\
M \pm S D \\
(95 \% \mathrm{Cl})\end{array}$ & $\begin{array}{l}\text { Sex } \\
F(p) \\
\omega_{p}^{2} \text { (effect) }\end{array}$ & $d$ (effect) \\
\hline \multirow{5}{*}{$\operatorname{Imp}[>10 \mathrm{G}]$} & Under-16 & $\begin{array}{l}5.98 \pm 4.14 \\
(4.87-7.09)\end{array}$ & $\begin{array}{l}2.66 \pm 3.22 \\
(1.80-3.51)\end{array}$ & \multirow{3}{*}{$\begin{array}{l}27.97 \text { (<.001) } \\
0.08 \text { (moderate) }\end{array}$} & 0.91 (moderate) \\
\hline & Under-18 & $\begin{array}{l}3.23 \pm 2.98 \\
(2.36-4.09)\end{array}$ & $\begin{array}{l}2.45 \pm 3.01 \\
(1.61-3.29)\end{array}$ & & 0.26 (low) \\
\hline & Senior & $\begin{array}{l}4.46 \pm 4.56 \\
(3.30-5.62)\end{array}$ & $\begin{array}{l}2.27 \pm 2.96 \\
(1.46-3.09)\end{array}$ & & 0.58 (low) \\
\hline & $\begin{array}{l}F(p) \\
\omega_{p}^{2}(\text { effect })\end{array}$ & $\begin{array}{l}4.72(.01) \\
0.02 \text { (low) }\end{array}$ & & \multirow{3}{*}{$\begin{array}{l}\text { Interaction } \\
3.36(.036) \\
0.01 \text { (low) }\end{array}$} & \\
\hline & post-hoc & A & & & \\
\hline \multirow{5}{*}{ Player load } & Under-16 & $\begin{array}{l}18.35 \pm 5.91 \\
(16.77-19.44)\end{array}$ & $\begin{array}{l}13.77 \pm 3.82 \\
(12.76-14.79)\end{array}$ & & 0.93 (moderate) \\
\hline & Under-18 & $\begin{array}{l}13.78 \pm 5.51 \\
(12.18-15.38)\end{array}$ & $\begin{array}{l}12.97 \pm 3.90 \\
(11.88-14.05)\end{array}$ & \multirow[t]{2}{*}{$\begin{array}{l}13.66(<.001) \\
0.04 \text { (low) }\end{array}$} & 0.17 (trivial) \\
\hline & Senior & $\begin{array}{l}15.05 \pm 5.69 \\
(13.60-16.49)\end{array}$ & $\begin{array}{l}14.35 \pm 4.43 \\
(13.13-15.57)\end{array}$ & & 0.14 (trivial) \\
\hline & $\begin{array}{l}F(p) \\
\omega_{p}^{2}(\text { effect })\end{array}$ & $\begin{array}{l}7.79(<.001) \\
0.04 \text { (low) }\end{array}$ & & \multirow{3}{*}{$\begin{array}{l}\text { Interaction } \\
5.49(.005) \\
0.03 \text { (low) }\end{array}$} & \\
\hline & post-hoc & $A, B$ & & & \\
\hline \multirow{5}{*}{ Steps } & Under-16 & $\begin{array}{l}935.43 \pm 344.37 \\
(843.20-1027.65)\end{array}$ & $\begin{array}{l}820.51 \pm 244.21 \\
(755.71-885.31)\end{array}$ & & 0.39 (low) \\
\hline & Under-18 & $\begin{array}{l}654.25 \pm 300.77 \\
(566.92-741.59)\end{array}$ & $\begin{array}{l}750.03 \pm 256.46 \\
(678.63-821.43)\end{array}$ & \multirow[t]{2}{*}{$\begin{array}{l}0.49(.486) \\
0.00\end{array}$} & -0.34 (low) \\
\hline & Senior & $\begin{array}{l}764.67 \pm 308.85 \\
(686.24-843.10)\end{array}$ & $\begin{array}{l}851.89 \pm 293.51 \\
(770.98-932.79)\end{array}$ & & -0.29 (low) \\
\hline & $\begin{array}{l}F(p) \\
\omega_{p}^{2}(\text { effect })\end{array}$ & $\begin{array}{l}9.57 \text { (<.001) } \\
0.05 \text { (low) }\end{array}$ & & \multirow{3}{*}{$\begin{array}{l}\text { Interaction } \\
4.56(.011) \\
0.02 \text { (low) }\end{array}$} & \\
\hline & post-hoc & $A, B$ & & & \\
\hline \multirow{5}{*}{ Jumps } & Under-16 & $\begin{array}{l}5.12 \pm 3.01 \\
(4.32-5.93)\end{array}$ & $\begin{array}{l}2.48 \pm 2.49 \\
(1.81-3.14)\end{array}$ & & 0.96 (moderate) \\
\hline & Under-18 & $\begin{array}{l}4.03 \pm 2.60 \\
(3.27-4.78)\end{array}$ & $\begin{array}{l}2.71 \pm 2.42 \\
(2.04-3.38)\end{array}$ & \multirow[t]{2}{*}{$\begin{array}{l}30.37(<.001) \\
0.08 \text { (moderate) }\end{array}$} & 0.53 (low) \\
\hline & Senior & $\begin{array}{l}5.67 \pm 4.63 \\
(4.50-6.85)\end{array}$ & $\begin{array}{l}3.74 .3 .31 \\
(2.83-4.65)\end{array}$ & & 0.49 (low) \\
\hline & $\begin{array}{l}F(p) \\
\omega_{p}^{2} \text { (effect) }\end{array}$ & $\begin{array}{l}4.89(.008) \\
0.02 \text { (low) }\end{array}$ & & \multirow{2}{*}{$\begin{array}{l}\text { Interaction } \\
1.14(.321) \\
0.00\end{array}$} & \\
\hline & post-hoc & & B & & \\
\hline
\end{tabular}

Note. M: mean; SD: standard deviation; Cl: confidence interval; p: p-value; d: Cohen's d effect size; F: ANOVA's Fvalue; $\omega_{p}^{2}$ : partial omega squared. Post-hoc: A Statistical differences between Under-16 and Under-18 $(p<0.05)$; B Statistical differences between Under-16 and senior $(p<0.05)$; C Statistical differences between Under-18 and senior $(p<0.05)$.

\section{DISCUSSION}

The analysis of the player's workload demands during matches is essential to establish the characteristics of the competition ${ }^{11}$. For this reason, the purpose of the present study was to analyze the gender-related and category-related differences in anthropometric characteristics and external workload demands during the competition. The main results showed that males obtained higher anthropometric values than females in height and weight in the three age categories (Under-16, Under-18 y senior). In addition, attending the BMI, there are significant differences only in the senior category with greater values in males. Respect to external workload 
demands males reported a greater number of impacts and jumps during matches, which results in a higher PL than females in all categories. These data show that the anthropometric profile has a direct influence on the external workload in handball, and also it produces differences in the external workload profile between genders ${ }^{25,26}$.

The anthropometric characteristics, age, and gender highly determine the requirements of talent selection in youth handball ${ }^{27}$. In the beach handball, there are great gender-related differences in height, weight, and body mass index (BMI). The present study shows that males obtained greater results in height and weight regardless of the age of the participant. However, there are only differences in the senior category with higher values in males in the BMI. Respect to the age and category of the participant, it is shown that weight and BMI increase in males, a symptom of the subject's own growth. In contrast, in women, it is observed that weight and BMI decrease as the category or age increases, while height increases according to human growth.

Previous research in handball has found similar results to those of the present study ${ }^{25,26}$, with higher values in males compared to females, and with respect to the increase in age, overall at the end of the maturing process. This aspect has been considered for talent detection. Mohamed et al. ${ }^{27}$ find that the greatest differences between elite and non-elite athletes were the effect of age and maturation on body mass, height, power and strength tests. Other research-based on handball also found differences in biological maturation, anthropometry and physical performance depending on the playing positions in handball ${ }^{28}$.

Another aspect to be highlighted in relation to the age of the athletes is that the females in the senior category were younger than men. This fact could indicate that women usually leave playing sports competition earlier than men ${ }^{29}$. Finally, it is possible to determine that the anthropometric profile of a beach handball player in the Under-16 category regardless of gender is around $63 \mathrm{~kg}$ in weight, $166 \mathrm{~cm}$ in height and $23 \mathrm{~kg} / \mathrm{m}^{2}$ in BMI. In the Under-18 category presented $64 \mathrm{~kg}$ of weight, $170 \mathrm{~cm}$ of height and $22 \mathrm{~kg} /$ $\mathrm{m}^{2}$ of BMI. While in the senior category, the anthropometrical profile was established around $70 \mathrm{~kg}$ of weight, $173 \mathrm{~cm}$ of height and $23 \mathrm{~kg} / \mathrm{m}^{2}$ of BMI.

The high-intensity intermittent actions have a great influence on the decision-making of beach handball players ${ }^{8}$. So, it is necessary to analyze and interpret in the most reliable way possible the different variables that explain these types of actions and establish the competition demands ${ }^{9}$. For this, EPTS called WIMU ${ }^{\mathrm{TM}}$ has been used to determine in an accurate way the movements performed by the players during the competition ${ }^{16}$. The knowledge, monitoring, and analysis of the workload allow to adapt the specific demands of the competition and determine the specific performance of each player during $\mathrm{it}^{20}$. Besides, it will be able to know with more precision the fatigue accumulated with the aim to design injury prevention and recovery programs $\mathrm{s}^{30}$, especially in congested-fixture tournaments as analyzed in the present study.

Different studies have analyzed the internal and external workload in handball both in players ${ }^{18,21}$, and referees ${ }^{20}$. Instead, only previous research 
analyzed the workload profile in beach handball ${ }^{8}$. This study showed a between-genders comparison at national and international-level players. The results obtained in the present research are similar to those obtained by Pueo et $\mathrm{al}^{8}$. Players usually performed low-intensity impacts (5-6 G) during the match with short periods of high-intensity activity that occurs with throws, stops, and counterattacks ${ }^{8}$. In the present study, the impact values were even lower (2-6 G). In the between-genders comparison, higher demands were performed by males in impacts, jumps and player load respect to females, as well as a greater volume of demands. In this sense, the investigations realized by Pueo et al. ${ }^{8}$ and Zapardiel and Asín-Izquierdo ${ }^{19}$ shown similar results in both genders related to the volume of displacements and actions, but higher demands in high-intensity actions in males respect to female beach handball players (high intensity impacts, collisions and high-intensity accelerations and decelerations).

Another aspect to consider into the competitive demands is the age category of the competition. These workload demands are different between regional, national and international-level matches, even between senior and youth players. The results of the present study shown that the under-16 category performed higher intensity and volume of impacts, jumps and player load respect to under-18 and senior category. These data could explain due to in under- 16 category does not exist any control of the match ${ }^{21}$ and players performed unnecessary displacements/skills that provoke over-efforts. These aspects over time with more understanding of the competition dynamics and better efforts control caused that the demands were reduced in under-18 and senior categories ${ }^{30}$. Therefore, an analysis of workload demands during official competition allows the establishment of individual training programs related to age category. These specific programs prepare handball players better for the competition scenarios for achieving higher probabilities of success in decision-making and physical performance ${ }^{10}$.

While the results of the present study shown an influence of gender and age category in anthropometric characteristics and external workload demands in beach handball players, through using inertial devices and giving an ecological treatment to research, there are different limitations that must be taken into consideration. The participants in the study had specific characteristics (amateur athletes of regional level), so the data should not be extrapolated to other populations of ages and different sports level. Furthermore, the data registering was made during a concentration format championship, with a large volume of matches (one match every one hour and a half) and in a covered installation with specific climatic conditions, therefore the results are specific to the competition format and the environmental conditions.

\section{CONCLUSION}

Category and gender have a direct influence on anthropometric characteristics in athletes. Males were older and with higher height and weight in every analyzed category. Regarding the category, an older age had higher values of 
height, weight, and BMI in athletes in both sexes. Therefore, it is important to consider these characteristics for the design of different aspects of performance such as basic physical abilities, nutrition or recovery processes, among others.

In addition, the age category and gender influenced the external load demands supported during the competition. Under-16 players performed the highest demands in relation to senior and under-18 players. The females presented lower workload demands in all categories regarding male players of the same age category, with special emphasis on the Under-16 category. All these aspects are essential to take them into consideration for training load planning and the design of training tasks in the sport modality of beach handball.

\section{COMPLIANCE WITH ETHICAL STANDARDS}

\section{Funding}

The author Carlos D. Gómez Carmona was supported by a grant from the Spanish Ministry of Innovation, Science and Universities (FPU17/00407). This study was co-funded by the Regional Department of Economy and Infrastructure of the Government of Extremadura (Spain) through the European Funds of Regional Development of the European Union (dossier number: GR18170).

\section{Ethical Aspects}

Ethical approval was obtained by the Bioethics Committee of the University of Extremadura (number 67/2017) and the protocol was written in accordance with standards set by the Declaration of Helsinki.

\section{Conflict of interests}

This work has been developed into the Research Group in Optimization of Training and Sports Performance (GOERD) of the Sports Science Faculty of the University of Extremadura. The funders had no role in the design of the study; in the collection, analyses, or interpretation of data; in the writing of the manuscript, or in the decision to publish the results. None of the authors has a conflict of interest to declare, and all authors were involved in the study design, data collection, and interpretation, and contributed to the writing of the manuscript.

\section{Contribution from authors}

Developed and coordinated the study: SJI and AA; Helped in data collection: CDG-C, DM-T and DG-S; Data analysis: CDG-C and DM-T; Wrote the article CDG-C, DM-T, DG-S, AA and SJI; Critical review of the article: AA and SJI

\section{REFERENCES}

1. Sena JEA, Gomes ALM, Mimbacas A, Ferreira UMG. Dermatoglifia, somatotipo e composição corporal no beach handball: Estudo comparativo entre diferentes níveis de qualificação esportiva. Motricidade. 2012;8(S2):567-76. 
2. International Handball Federation. History of beach handball. Basel, Switzerland: International Handball Federation; 2010.

3. Achenbach L, Loose O, Laver L, Zeman F, Nerlich M, Angele P, et al. Beach handball is safer than indoor team handball: injury rates during the 2017 European Beach Handball Championships. Knee Surg Sports Traumatol Arthrosc. 2018;26(7):1909-15.

4. Real Federación Española de Balonmano. Reglas de Juego - Balonmano Playa. 1a Edición. Madrid, España: Real Federación Española de Balonmano; 2014. 127 p.

5. Morillo-Baro JP, Reigal RE, Hernandez-Mendo A. Analysis of positional attack in beach handball male and female with polar coordinates. RICYDE Rev Int Cienc Deporte. 2015;11(41):226-44.

6. Gruić I, Vuleta D, Bazzeo M, Ohnjec K. Situational efficiency of teams in female part of tournement in the World Beach Handball Championship in Cadiz. 6th International Scientific Conference on Kinesiology, Croatia; 2011. p. 524-8.

7. Navarro A, Morillo JP, Reigal RE, Hernández-Mendo A. Polar coordinate analysis in the study of positional attacks in beach handball. Int J Perform Anal Sport. 2018;18(1):151-67.

8. Pueo B, Jimenez-Olmedo JM, Penichet-Tomas A, Becerra MO, Agullo JJE. Analysis of Time-Motion and Heart Rate in Elite Male and Female Beach Handball. J Sport Sci Med. 2017;16:450-8.

9. Belka J, Hulka K, Safar M, Weisser R, Samcova A. Analyses of time-motion and heart rate in elite female players (u19) during competitive handball matches. Kinesiology. 2014;46(1):33-43.

10. Lara-Cobos D. La respuesta cardiaca durante la competición de balonmano playa femenino. Apunts Med Esport. 2011;46(171):131-6.

11. Bartlett JD, O'Connor F, Pitchford N, Torres-Ronda L, Robertson SJ. Relationships Between Internal and External Training Load in Team-Sport Athletes: Evidence for an Individualized Approach. Int J Sports Physiol Perform. 2017;12(2):230-4.

12. Achten J, Jeukendrup AE. Heart rate monitoring. Sports Med. 2003;33(7):517-538.

13. Bastida-Castillo A, Gómez-Carmona CD, Hernandez A, Pino-Ortega J. Validezy fiabilidad de un dispositivo inercial (WIMU PROTM) para el análisis del posicionamiento en balonmano. E-Balonmano Com Rev Cienc Deporte. 2018;14(1):9-16.

14. Bastida Castillo A, Gómez Carmona CD, De la Cruz Sánchez E, Pino Ortega J. Accuracy, intra- and inter-unit reliability, and comparison between GPS and UWB-based position-tracking systems used for time-motion analyses in soccer. Eur J Sport Sci. 2018;18(4):450-7.

15. Bastida-Castillo A, Gómez-Carmona CD, de la Cruz Sánchez E, Pino-Ortega J. Comparing Accuracy between Global Positioning Systems and Ultra-WidebandBased Position Tracking Systems Used for Tactical Analyses in Soccer. EurJ Sport Sci. 2019; Epub: Ahead of print.

16. Gómez-Carmona CD, Bastida-Castillo A, García-Rubio J, Ibáñez SJ, Pino-Ortega J. Static and dynamic reliability of WIMU PROTM accelerometers according to anatomical placement. Proc Inst Mech Eng Part P J Sports Eng Technol. 2019;233(2):238-48.

17. Karcher C, Buchheit M. On-Court Demands of Elite Handball, with Special Reference to Playing Positions. Sports Med. 2014;44(6):797-814.

18. Michalsik LB, Aagaard P. Physical demands in elite team handball: Comparisons between male and female players. J Sports Med Phys Fitness. 2015;55(9):878-891.

19. Zapardiel JC, Asín-Izquierdo I. Conditional analysis of elite beach handball according to specific playing position through assessment with GPS. Int J Perform Anal Sport. 2020; Epub: Ahead of print.

20. García-Santos D, Gamonales JM, León K, Mancha-Triguero D, Muñoz-Jiménez J. Un estudio de casos: caracterización de las demandas fisiológicas, cinemáticas y neuromusculares de un árbitro de balonmano. E-Balonmano Com Rev Cienc Deporte. 2017;13(3):207-16. 
21. Mancha-Triguero D, Reina M, Baquero B, García-Rubio J, Ibáñez SJ. Análisis de la carga competitiva en jugadores de balonmano de formación en función del resultado final. E-Balonmano Com Rev Cienc Deporte. 2018;14(2):99-108.

22. Gómez-Carmona CD, Pino-Ortega J, Sánchez-Ureña B, Ibáñez SJ, RojasValverde D. Accelerometry-Based External Load Indicators in Sport: Too Many Options, Same Practical Outcome? Int J Environ Res Public Health. 2019 Dec 13;16(24):5101.

23. Field A. Discovering Statistics Using IBM SPSS Statistics. 4th ed. Londres: SAGE; 2013. 954 p.

24. Cohen J. Statistical power analysis for the behavioral sciences. 2nd ed. Hillsdale, NJ: Erlbaum; 1988.

25. Zapartidis I, Palamas A, Papa M, Tsakalou L, Kotsampouikidou Z. Relationship among anthropometric characteristics, handgrip strength and throwing velocity in adolescent handball players. J Phys Educ Sports Manag. 2016;3(1):127-139.

26. Grijota Pérez FJ, Montero Arroyo J, Llerena Ruiz F, Muñoz Marín D. Análisis Comparativo de la Composición Corporal y de la Condición Física en Jóvenes Practicantes de Balonmano. Kronos Rev Entren Deport. 2015;29(2):21-27.

27. Mohamed H, Vaeyens R, Matthys S, Multael M, Lefevre J, Lenoir M, et al. Anthropometric and performance measures for the development of a talent detection and identification model in youth handball. J Sports Sci. 2009;27(3):257-266.

28. Ziv G, Lidor R. Physical characteristics, physiological attributes, and on-court performances of handball players: A review. Eur J Sport Sci. 2009;9(6):375-86.

29. Carlin M, Salguero A, Rosa SM, de los Fayos EJG. Análisis de los motivos de retirada de la práctica deportiva y su relación con la orientación motivacional en deportistas universitarios. Cuad Psicol Deporte. 2009;9(1):85-99.

30. Colby MJ, Dawson B, Heasman J, Rogalski B, Gabbett TJ. Accelerometer and GPS-Derived Running Loads and Injury Risk in Elite Australian Footballers: J Strength Cond Res. 2014;28(8):2244-52.

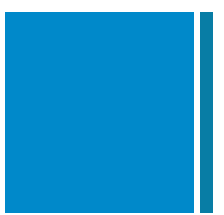

Corresponding author

Carlos D. Gómez-Carmona

Training Optimization and Sports Performance Research Group (GOERD)

Faculty of Sports Sciences, University of Extremadura, Cáceres, Spain.

Email: cdgomezcarmona@unex.es 